\title{
Structural and Functional Changes across the Visual Cortex of a Patient with Visual Form Agnosia
}

\author{
Holly Bridge, ${ }^{1 \star}$ Owen M. Thomas, ${ }^{2 \star}$ Loredana Minini, ${ }^{3}$ Cristiana Cavina-Pratesi, ${ }^{5}$ A. David Milner, ${ }^{5}$ and \\ Andrew J. Parker ${ }^{4}$ \\ ${ }^{1}$ Functional MRI of the Brain Centre, Nuffield Department of Clinical Neurosciences, John Radcliffe Hospital, Oxford, OX3 9DU, United Kingdom, \\ ${ }^{2}$ University Department of Radiology, Addenbrookes Hospital, Cambridge, CB2 2QQ, United Kingdom, ${ }^{3}$ Department of Experimental Psychology, \\ University of Oxford, Oxford, OX1 3UD, United Kingdom, ${ }^{4}$ Department of Physiology, Anatomy, and Genetics, University of Oxford, Oxford, OX1 3PT, \\ United Kingdom, and ${ }^{5}$ Department of Psychology, Durham University Science Site, Durham, DH1 3LE, United Kingdom
}

Loss of shape recognition in visual-form agnosia occurs without equivalent losses in the use of vision to guide actions, providing support for the hypothesis of two visual systems (for "perception" and "action"). The human individual DF received a toxic exposure to carbon monoxide some years ago, which resulted in a persisting visual-form agnosia that has been extensively characterized at the behavioral level. We conducted a detailed high-resolution MRI study of DF's cortex, combining structural and functional measurements. We present the first accurate quantification of the changes in thickness across DF's occipital cortex, finding the most substantial loss in the lateral occipital cortex (LOC). There are reduced white matter connections between LOC and other areas. Functional measures show pockets of activity that survive within structurally damaged areas. The topographic mapping of visual areas showed that ordered retinotopic maps were evident for DF in the ventral portions of visual cortical areas V1, V2, V3, and hV4. Although V1 shows evidence of topographic order in its dorsal portion, such maps could not be found in the dorsal parts of V2 and V3. We conclude that it is not possible to understand fully the deficits in object perception in visual-form agnosia without the exploitation of both structural and functional measurements. Our results also highlight for DF the cortical routes through which visual information is able to pass to support her well-documented abilities to use visual information to guide actions.

\section{Introduction}

Individuals who have suffered damage to specific regions of the cortex have long contributed to understanding the specialization of the human brain. In the majority of cases, damage is unilateral, with only a modest impact on visual perception. Occasionally, there are cases of bilateral damage to corresponding regions in the two hemispheres that can profoundly affect specific aspects of visual perception.

An incident of carbon monoxide poisoning in patient DF caused bilateral damage to regions of the occipital cortex, resulting in a severe visual-form agnosia in addition to prosopagnosia (Goodale et al., 1991; Milner et al., 1991; Milner, 1997; James et al., 2003). Color, motion, and texture perception remain relatively intact (Cavina-Pratesi et al., 2010a). DF's behavior demonstrates an important feature of the visual nervous system, which is that vision can be used for information about objects to control actions in the absence of object recognition. This observation

\footnotetext{
Received Oct. 3, 2012; revised June 11, 2013; accepted June 21, 2013.

Author contributions: H.B., C.C.P., A.D.M., and A.J.P. designed research; H.B. and L.M. performed research; H.B., O.M.T., and L.M. analyzed data; H.B., C.C.-P., A.D.M., and A.J.P. wrote the paper.

This work was supported by Medical Research Council Grants G0401090 (A.D.M.) and G0802171 (H.B., A.J.P.), a Royal Society University Research Fellowship (H.B.), and a Royal Society Wolfson Merit Award (A.J.P.).

*H.B. and O.M.T. contributed equally to this work.

Correspondence should be addressed to Andrew J. Parker, Department of Physiology, Anatomy, and Genetics, Sherrington Building, Parks Road, 0xford, 0X1 3PT, UK. E-mail: andrew.parker@dpag.ox.ac.uk.

DOI:10.1523/JNEUROSCI.4853-12.2013

Copyright $\odot 2013$ the authors $\quad 0270-6474 / 13 / 3312779-13 \$ 15.00 / 0$
}

provides support for the hypothesis of parallel visual streams for perception and action (Castiello and Jeannerod, 1991; Goodale and Milner, 1992; Milner, 1997). Specifically, although object identification, a ventral stream function, is devastatingly impaired in DF, reaching and grasping for objects, supported by the dorsal stream, appear relatively normal both behaviorally and with functional MRI (James et al., 2003).

Brain damage caused by carbon monoxide poisoning is typically widespread (O'Donnell et al., 2000); a previous MRI study has indicated this in DF (James et al., 2003). On the basis of previous analyses of MRI and DF's behavior, it has been proposed that DF's ventral stream processing is interrupted by bilateral lesions of lateral occipital cortex (LOC). Recently, this association has been questioned; Karnath et al. (2009) proposed that ventromedial areas of occipito-temporal cortex are more significant based on the location of a bilateral stroke, also resulting in agnosia. Here, we examined gray and white matter integrity in all occipital areas (lateral and medial) of DF's cortex to establish whether the damage extends into the early visual cortex, whether it is specific to higher visual areas, and, if so, which areas are most seriously compromised. Accurate identification of locations of damage in DF's brain will assist in understanding how the condition of visual agnosia arises.

Complementary to these structural measures, retinotopic mapping allows the functional identification of early visual areas and also certain higher visual areas (Wandell and Winawer, 2011). Applying this technique to DF's visual system delineates 
precisely the visual field topography of functional activity. Finally, given the recent demonstration of selective problems in stereoscopic depth perception in DF (Read et al., 2010), we also investigated the pattern of functional activations to a disparitydefined stimulus, as well as a collection of other "localizer" stimuli, specifically designed to test cortical functions related to object recognition, visual motion detection, and chromatic signaling.

\section{Materials and Methods}

Participants. The study was conducted under ethical approval from the Oxfordshire National Health Service Research Ethics Committee (08/ H0605/156), and all participants provided informed written consent. Patient DF has been extensively described previously (Milner et al., 1991; James et al., 2003): at the time that these data were acquired, she was 55 years old. Eight participants were recruited as control subjects specifically for this study, of whom two were age matched to DF and six were younger (mean, $29 \pm 6$ years; three females), more similar to the age of DF (34 years) at the time of the original accident. All structural and functional MRI data were acquired in these eight control subjects. In addition, data from three additional age-matched controls were available for T1weighted, diffusion-weighted MRI and motion-activated functional MRI experiments, and an additional age-matched control was used for cortical thickness analysis. For the cortical thickness analysis, only the six age-matched controls were used (mean, $61 \pm 9$ years; two females, four males). Similarly, diffusion-weighted analysis and motion processing were performed on the five available age-matched controls only (mean, $61 \pm 6$ years; two females, three males), as well as on the group of eight younger control subjects to account for age-related effects.

Data acquisition. Scanning was performed at the Oxford Centre for Clinical Magnetic Resonance using a Siemens $3 \mathrm{~T}$ Trio scanner with a 12-channel head coil. For control participants, data were acquired in a single scanning session, consisting of a T1-weighted structural scan, a diffusion-weighted scan, and four functional MRI scans. Data from DF were acquired over $2 \mathrm{~d}$, with $1 \mathrm{~d}$ consisting of acquisition of the scans in the control participants and an additional session in which retinotopy data were collected.

The T1-weighted structural scan was performed with a spatial resolution of $1 \times 1 \times 1 \mathrm{~mm}^{3}$, using standard parameters [magnetization prepared rapid gradient echo; repetition time (TR), $15 \mathrm{~ms}$; echo time (TE), $6.0 \mathrm{~ms}$. Diffusion-weighted images were acquired axially using echo-planar imaging (EPI), with isotropic voxels of $2 \mathrm{~mm}^{3}$. The diffusion weighting was isotropically distributed through space (Bondok and Sansone, 1984) along 60 directions using a $b$-value of $1000 \mathrm{~s} / \mathrm{mm}^{2}$. Two sets of diffusion-weighted data were collected, and, for each set, five volumes with no diffusion weighting were acquired during the sequence.

Visual stimuli for $\mathrm{fMRI}$ were presented using a $100 \mathrm{~Hz}$ Christie Mirage stereoscopic projector attached to a $z$-screen that applied opposite circular polarization to the light forming the image projected to each eye. Participants lay supine in the scanner while wearing polarizing glasses (and any necessary refractive correction) and viewed the screen through a mirror above the eyes to make a binocular display. For the binocular disparity experiment, this polarizing system permitted the presentation of different stimuli independently to each eye, with minimal crosstalk.

Retinotopic mapping of cortical areas. Retinotopic data were acquired coronally from DF in a separate session with an EPI sequence (TE, $30 \mathrm{~ms}$; TR, $4000 \mathrm{~ms}$; 562 -mm slices; $2 \times 2 \mathrm{~mm}$ in-plane resolution; matrix, $64 \times$ 64). Each run consisted of 48 volumes acquired continuously (192 s). To assist in identifying human visual cortical area V5/middle temporal area MT, as well as other retinotopically defined areas, the mapping stimulus was a wedge consisting of 500 moving black dots on a white background. The apex of the wedge was at the fixation point. This wedge subtended $90^{\circ}$ and rotated $45^{\circ}$ every TR (4s). The dots within the wedge moved along radial trajectories inward and outward, changing direction every $1 \mathrm{~s}$, as described previously (Bridge and Parker, 2007). Each run consisted of six complete cycles of the wedge. Eccentricity maps were generated using expanding concentric rings at eight different eccentricities, to a maximum eccentricity of $12^{\circ}$. The rings consisted of a black-and-white dartboard stimulus, flickering at $8 \mathrm{~Hz}$. The rings were presented for $4 \mathrm{~s}$ in each position before advancing to the next position, such that a single cycle took $48 \mathrm{~s}$, and six cycles were presented in total.

This retinotopy session also included a reduced field of view (40 2-mm slices) T1-weighted image (three-dimensional fast, low-angle shot sequence), acquired coronally at an in-plane resolution of $1 \times 1 \mathrm{~mm}^{2}$. These slices were in the same planes as the functional images and were used as an intermediate step to register the retinotopy data to the wholebrain anatomy.

Polar angle maps were extracted with the mrVista package (http://white.stanford.edu/newlm/index.php/Software). This software was also used for motion correction and registration of the functional images to the anatomy. The resulting maps were displayed on flat renderings of the occipito-temporo-parietal region on which the borders between the visual areas were identified.

$f M R I$ responses to disparity, motion, color, and objects. A sequence of fMRI scans was used to characterize the response of DF's cortex to the disparity, motion, color, and form of objects. Scans were acquired in DF and control participants at a resolution of $2.8 \times 2.8 \times 2.8 \mathrm{~mm}^{3}$, with a TR of $3 \mathrm{~s}$ for the disparity experiment and $4 \mathrm{~s}$ for the motion, object, and color scans.

The first fMRI scan measured the response to changing disparity. The stimulus $\left(16^{\circ} \times 16^{\circ}\right.$ square $)$ consisted of black-and-white dots on a midgray background. In the "zero-disparity" condition, all dots were matched for position in the two eyes. In the "changing-disparity" condition, the inner portion of the plane of dots $\left(12^{\circ} \times 12^{\circ}\right)$ was divided into nine squares, each $4^{\circ} \times 4^{\circ}$ to which a horizontal disparity was allocated at random from the range of $-0.3^{\circ}$ to $0.3^{\circ}$. The randomly allocated disparity changed in value every second to give a stimulus that appeared to be changing in depth. Each block consisted of $15 \mathrm{~s}$ of zero disparity alternated with $15 \mathrm{~s}$ of changing disparity. Ten blocks were presented in each scan, giving a total scan length of $300 \mathrm{~s}$. This scan was performed twice in all participants.

The motion scan consisted of two alternating blocks of "moving dots" and "stationary dots." An $8^{\circ}$ diameter patch of black square dots $\left(0.5^{\circ} \times\right.$ $0.5^{\circ}$ ) was presented on a white background. During the moving block, the dots moved radially at a speed of $30 \%$, reversing direction every second. A complete cycle of stimulus (on and off) lasted $32 \mathrm{~s}$, and eight such cycles were presented in total. In patient DF and five control participants, we performed two runs of the experiment, which were combined using a fixed-effects analysis.

To determine the cortical response to objects and color, a scan was performed in which five different blocks were presented. An "object" block consisted of 16 different stimuli presented in color. The "scrambled object" block consisted of the same stimuli but scrambled in phase (Malach et al., 1995). The "color" block consisted of 16 Mondrian type patterns, and the "black-and-white" block had the same Mondrian patterns but without the color information. In each case, the stimuli making up the block were presented for $1 \mathrm{~s}$ each. A "rest" block lasting $16 \mathrm{~s}$ consisted of a fixation cross on a uniform background. Therefore, the full cycle of blocks was 80 s long; each cycle was presented five times.

Data analyses. Analysis of cortical thickness was performed using Freesurfer (Dale et al., 1999) (http://surfer.nmr.mgh.harvard.edu). This method has been described and validated previously (Fischl and Dale, 2000). The critical steps for calculating cortical thickness are the segmentation of the white matter, the tessellation of the white matter boundary, smoothing of this surface, and automated topology correction. The white matter surface is then deformed outward to find the pial surface. Cortical thickness is then computed as the shortest distance between the two surfaces by taking the mean of the shortest distance computed from the white matter to pial surface and in reverse from the pial surface to the white matter. Values are generated for every vertex on the white matter surface, which can then be averaged across all the vertices within a specific region of interest. Mean cortical thickness was extracted from individual visual areas from the retinotopy-defined atlas described previously. The probabilistic definitions of these areas were thresholded at $30 \%$ (i.e., such that the area was defined in at least $30 \%$ of subjects) and then binarized. The areas were then transformed onto the Freesurfer cortical surface for each individual participant, using the mri_surf2vol command. 
LOC thickness was extracted using the overlap of the functional definition derived from comparing colored pictures of objects with scrambled versions of those objects. This may define an area slightly more extensive than the core LOC, which has been defined previously on the basis of comparing achromatic, black-and-white line drawings with scrambled versions of those drawings (James et al., 2003). As nonvisual control regions, Brodmann areas $3 \mathrm{~b}$ and $4 \mathrm{a}$ were extracted from the Freesurfer atlas (Fischl et al., 2004). These areas are defined within the atlas by postmortem cytoarchitecture and were identified in individual participants in this study based on cortical folding patterns.

To investigate the cortical thickness of the ventromedial occipital cortex, definitions of the fusiform and lingual gyri were extracted from the Harvard-Oxford cortical structural atlas in FSL [for FMRIB Software Library, in which FMRIB is Functional MRI of the Brain, Oxford University, Oxford, UK)], summed, and transformed into the structural space of each individual subject. Similarly, the inferior LOC definition was extracted from the same atlas to be used as a ventrolateral region mask. These masks were thresholded and binarized before being projected onto the Freesurfer cortical surface such that the cortical thickness could be extracted individually for each subject.

Functional data were analyzed using FEAT (for FMRIB fMRI Expert Analysis Tool), part of the FSL toolbox (www.fmrib.ox.ac.uk). Images were preprocessed using a number of steps: head movement correction using MCFLIRT (for FMRIB Linear Image Restoration Tool with Motion Correction), spatial smoothing with a Gaussian kernel full-width at half-maximal of $5 \mathrm{~mm}$, mean-based intensity normalization, and nonlinear high-pass temporal frequency filtering (Gaussian-weighted straight line fitting, with $\sigma=30 \mathrm{~s}$ ). In the case of the disparity experiment, data from the two scans were combined using a fixed-effects analysis. Data from control participants were combined using FLAME (for FMRIB Local Analysis of Mixed Effects) to produce group activation maps.

Visual areas for control participants were defined using an atlas based on 16 healthy control participants, on whom retinotopic mapping had been performed (Bridge, 2011). Because the resulting maps were probabilistic, each visual area was thresholded such that there was no overlap between adjacent visual areas. Area LOC was extracted using the activation data of the control participants transformed into Montreal Neuroscience Institute (MNI) space: a voxel was considered to lie within LOC if the activation to objects compared with scrambled objects exceeded a $z$-transformed value of 2 , in six of the eight control participants.

Probabilistic tractography produces an estimate of the most likely location of a pathway from a seed point using Bayesian techniques, as described previously (Behrens et al., 2007). A local model for fiber orientation within each voxel is inferred from the data. Probabilistic tractography consists of the construction of pathways by following sample orientations in each voxel along the trajectory. From each seed voxel within the seed mask, 5000 sample tracts were generated, and tracts entering the target masks were retained. To ensure that the pathways were restricted in extent, an exclusion mask was incorporated to prevent projections crossing into the contralateral hemisphere. Quantitative measures of fractional anisotropy (FA) and mean diffusion for individual participants were extracted by taking the fiber-density-weighted mean within each of the tract.

To determine whether the data from DF differed quantitatively from the control group data, we used the method of Crawford et al. (2010), specifically designed for comparing an individual's test score against norms derived from small samples. Because this is a modified $t$ test, we refer to this as a Crawford, Garthwaite, Porter modified (CGP-modified) $t$ test elsewhere in the paper.

\section{Results}

Our results are separately presented as structural and functional assessments of the damage to and rearrangement of visual pathways within DF's occipital cortex. Damage to the cerebral cortex as a result of toxic agents (such as carbon monoxide) is inherently variable and a matter of degree rather than a clear-cut loss of some cortical tissue. Therefore, we applied quantitative measures of cortical structure and function at all stages of this analysis. In comparing different measures of cortical structure and function, these are overlaid on single representations of the cortical surface, either flat maps or inflated hemispheres, so that direct visual correspondence can be assessed.

\section{Quantification of structural damage across the cortical surface}

To quantify the damage to gray and white matter in DF, cortical thickness was measured in DF and six participants of a comparable or greater age (mean $\pm \mathrm{SD}, 61 \pm 9$ years). Figure 1 shows for DF and an age-matched control the map of cortical thickness on the inflated surface of their brains. Several regions of the occipital lobe of DF's brain stand out as having reduced cortical thickness, including the LOC (blue arrows) and the posterior intraparietal sulcus (IPS) (yellow arrows). Additionally, there is damage to the anterior portion of V1 in the left hemisphere, which can be seen in the medial view of DF's brain in the top row.

The bar charts show mean thickness of gray matter across the age-matched control participants and DF. A single asterisk indicates that the cortical thickness in DF is significantly lower at $p<0.05$, and a double asterisk indicates $p<0.01$, using the CGP-modified $t$ test from Crawford et al. (2010). The majority of occipital cortical areas are thinner in DF compared with the control participants; only V1 (control mean, $1.6 \pm 0.11 \mathrm{~mm}$; DF, 1.52 $\mathrm{mm}$ ) and MT (control mean, $2.35 \pm 0.16 \mathrm{~mm}$; DF, $2.06 \mathrm{~mm}$ ) do not significantly differ in thickness. Therefore, it is clear that the damage to DF's occipital cortex spreads considerably beyond LOC, which is generally considered the most affected region. Measurements presented below indicate differences in the ability of dorsal and ventral portions of $\mathrm{V} 2$ and $\mathrm{V} 3$ to generate functional activations, so the structural analysis of thickness presented here is also divided into dorsal and ventral segments of these areas (V2v, V2d, V3v, V3d).

As a control sample from nonvisual cortex, cortical thickness values from Brodmann areas $3 \mathrm{~b}$ and $4 \mathrm{a}$ were extracted from the Freesurfer thickness maps. In both of these areas, DF's cortex is slightly thicker than the control mean, and, in area $3 \mathrm{~b}$, it is significantly thicker than controls (control mean $\pm \mathrm{SE}, 1.75 \pm 0.06$ $\mathrm{mm}$; DF, $2.22 \mathrm{~mm} ;=3.7 ; p<0.05$, CGP-modified $t$ test). A larger group of younger controls $(n=12)$ from a separate study showed very similar thickness in area $3 \mathrm{~b}$ when compared with the older control group (mean $\pm \mathrm{SE}, 1.76 \pm 0.02 \mathrm{~mm}$ ).

Although the cortical thinning is obvious in LOC in DF, it is important to also consider the ventromedial occipital areas highlighted by Karnath et al. (2009) to evaluate whether these areas may be contributing to the agnosia. Figure 2 shows the regions of occipital cortex in left and right hemispheres that were investigated for this analysis, shown on DF's brain (top left). The bottom left of Figure 2 shows the cortical thickness on the ventral surface of the cortex in DF compared with the cortex of the ageand gender-matched control subject. The cortical thicknesses extracted from DF and the group of older control subjects do not differ, suggesting that the gray matter structure in this region is not greatly affected in DF. Certainly the thinning of ventromedial cortex in DF is considerably less than that for LOC and most of the early visual areas (V1 and MT excepted).

Formal statistical comparisons of the thickness of gray matter in DF against controls confirm this point. Subjecting the values for ventrolateral cortex to CGP-modified $t$ tests (Crawford et al., 2010) yields the following: left hemisphere, $t=-3.6$ with $p=$ 0.02 ; and right hemisphere, $t=-2.5$ with $p=0.06$. By comparison, for ventromedial cortex, the same tests yield the following: 


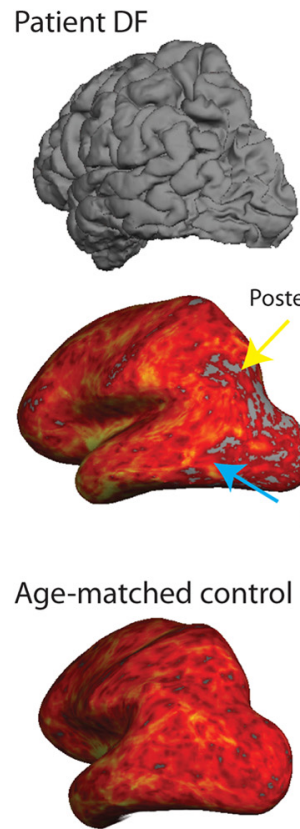

Left
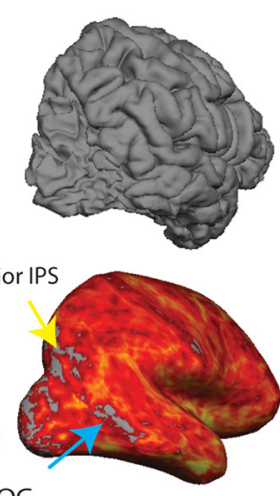

$\mathrm{OC}$
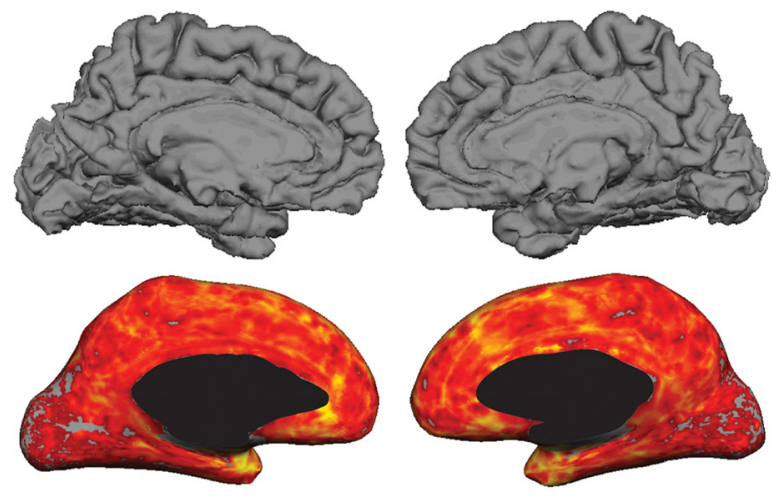

$.2 \mathrm{~mm}$
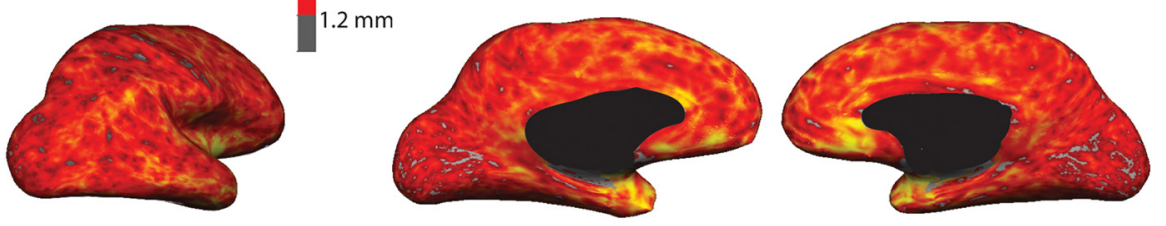

Right
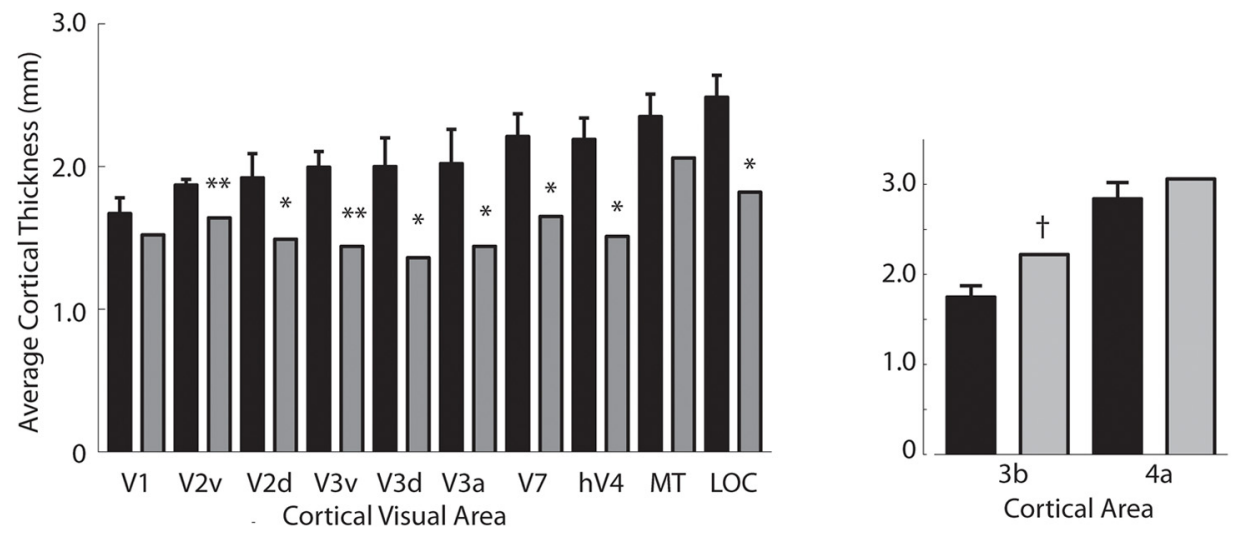

Figure 1. Cortical thickness is significantly reduced in extrastriate regions in DF compared with age-matched control participants. The top row shows the pial surface of DF's brain. Frontal and temporal regions appear to show normal gyrification, but the occipital cortex shows considerable reduction in gray matter, particularly on the lateral surface. The red scale for DF's brain in the second row indicates a quantification of the gray matter thickness. The arrows indicate areas LOC (cyan) and posterior IPS (yellow) in which the cortical thickness is $<1.2 \mathrm{~mm}$. Comparable data for an age-matched female control are shown below. The asterisks in the bottom left indicate visual cortical areas, for which the cortex in DF (gray bars) is significantly thinner than controls (black bars) across both hemispheres (using (GP-modified t tests from Crawford et al. (2010), with ${ }^{*} p<0.05$ and ${ }^{* *} p<0.01$; see also Table 1). Only areas V1 (right hemisphere only) and MT do not differ from control participants. Cortical thickness for dorsal and ventral regions of V2 and V3 (V2d, V2v, V3d, V3v) are shown separately for comparison with retinotopic mapping results in Figure 3. Brodmann area $3 \mathrm{~b}$, indicated by $\uparrow$ and used as a control region, is significantly thicker in DF than control participants.

left hemisphere, $t=-1.7$ with $p=0.16$; and right hemisphere, $t=-1.3$ with $p=0.24$ (all comparisons are two-tailed; see Table 1). Hence, in regard to cortical thickness in DF's brain, the ventrolateral cortex (including the LOC) is significantly affected, whereas the ventromedial cortex is not.

\section{Retinotopic mapping of visual areas in DF}

Standard retinotopic mapping in DF caused zones of activation time-locked to the rotation of the visual stimulus around the fixation point. Activation was evident in the ventral portion of the early visual cortex to both rotating wedge and expanding ring stimuli (Fig. 3). Ventral regions in DF were similar to control participants, with the predicted retinotopic organization of the visual field in areas V1, V2, V3, and hV4 (in the right hemisphere). In contrast, in the dorsal portions of visual cortex, retinotopically mapped activation was only evident in dorsal V1, with no apparent retinotopic mapping in dorsal V2 or V3.
DF was only available to participate in a single scanning session for retinotopic mapping. With additional sessions to boost the statistical power, a retinotopic organization may yet be discovered in dorsal parts of DF's V2 and V3. This might also permit the use of automated methods for delineating the retinotopic areas. The visual areas have been manually demarcated in this study, because the current activation patterns are unlikely to be sufficient for an automated algorithm. There is a possibility that any changes in vasculature as a result of the carbon monoxide poisoning may also have changed the hemodynamics of regions of the DF's visual cortex, such that the maps are inherently less clear. In a participant with blindsight, Radoeva et al. (2008) found a retinotopic organization within the scotoma but only after multiple sessions of retinotopic mapping. It must also be recognized that absence of a retinotopic map in a region of cortex does not imply that there is a complete absence of functional activity within that cortical region. 



Age-matched female control
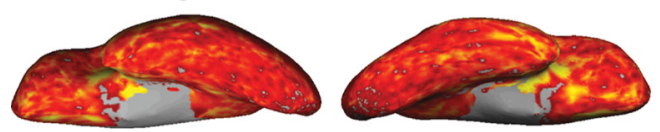

3.0

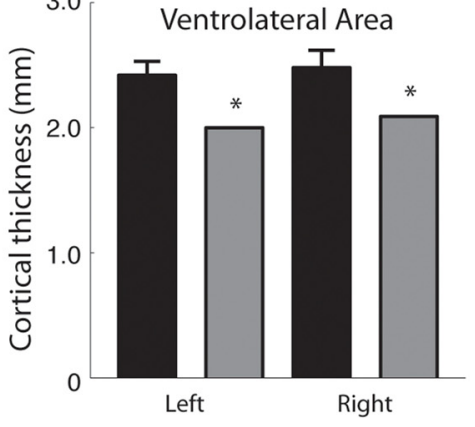

Figure 2. Cortical thickness in ventromedial and ventrolateral occipital cortex. Cortical thickness values for the ventromedial cortex were extracted from the masks shown on DF's brain (top left). Bottom left shows a comparison of the cortical thickness values on the ventral surface of DF's brain and that of the age-matched female control subject. Cortical thickness in DF in ventromedial cortex does not differ from controls (top right). Black bars, Controls; gray bars, DF. In ventrolateral cortex (bottom right), there is a significant reduction in cortical thickness in DF (CGP-modified $t$ tests from Crawford et al, 2010, with * $p=0.05$ ). See also Table 1.

Table 1. Statistical tests of differences in cortical thickness in DF's brain compared with controls

\begin{tabular}{lll}
\hline Area & CGP-modified $t$ statistic & Significance \\
\hline V1 & -1.24 & NS \\
V2d & -2.85 & $p<0.05$ \\
V2v & -6.36 & $p<0.005$ \\
V3d & -2.78 & $p<0.05$ \\
V3v & -4.40 & $p<0.01$ \\
V3a & -2.46 & $p<0.06$ \\
V7 & -3.20 & $p<0.05$ \\
V4 & -3.80 & $p<0.05$ \\
MT & -1.60 & NS \\
L0C & -4.08 & $p<0.05$ \\
3b & +3.40 & $p<0.05$ \\
4a & +1.12 & NS \\
Ventrolateral & -3.11 & $p<0.05$ \\
Ventromedial & -1.73 & NS \\
\hline
\end{tabular}

The CGP-modified $t$ test was specifically designed for comparing a single case study against a group of control participants (Crawford et al., 2010).

To examine whether the absence of retinotopy might be attributable to differences in the cortical thickness between the dorsal and ventral regions of the early visual cortex, the thicknesses were calculated separately for the ventral and dorsal divisions of V2 and V3 (Fig. 1). There was little difference between the cortical thicknesses of these divisions in controls (differences $<0.05 \mathrm{~mm}$ ). For DF, the values for V2 were $1.49 \mathrm{~mm}$ dorsal and $1.64 \mathrm{~mm}$ ventral, and for $\mathrm{V} 3$, the values were $1.36 \mathrm{~mm}$ dorsal and $1.44 \mathrm{~mm}$ ventral. To ensure that the lack of visible activation in the retinotopic mapping was not an artifact of the cortical depth at which the analysis had been performed, the activation patterns were additionally inspected in the native volume space. Inspection in volume space did not reveal any evidence for activations that might otherwise have been missed in the data analysis procedures for retinotopic mapping.

Equally, it is known that poor fixation behavior is capable of disrupting retinotopic mapping procedures, even in normal subjects. Nonetheless, DF's fixation behavior has recently been monitored again (Hesse et al., 2012), and there are no obvious differences between her ability to fixate and the ability of controls. Moreover, an explanation based purely on fixation errors does not readily account for why DF's results show evidence of retinotopy that can be identified in ventral regions of V2 and V3 but not in the equivalent dorsal regions of those areas. Furthermore, retinotopic organization is present in both dorsal and ventral regions of $\mathrm{V} 1$, which is another argument against an explanation based solely on fixation errors.

Referring back to previous results, it is worth noting that DF's visual thresholds were tested in April 1998 using Humphrey static perimetry (C.C.-P. and A.D.M., unpublished data). There is significantly reduced detection in the lower quadrants of both the right and left visual hemifields. In detail, there is a $10-15 \mathrm{~dB}$ difference in Humphrey pattern deviation measures at eccentricities of $12^{\circ}$. Measurements were taken separately for left and right eyes and then averaged to give a single value for the difference between upper and lower quadrants for left and right visual hemifields. Paired sample $t$ tests (one-tailed, corrected for multiple comparisons) showed that these sensitivity measurements in the lower field quadrants of DF are significantly lower than those for the upper field quadrants for both the right $(t=3.74 ; p=0.033)$ and left $(t=4.76 ; p=0.018)$ visual hemifields.

A reduced functionality of the dorsal portions of V2 and V3 could explain this observation given that these areas represent the lower halves of the visual field. In other words, it may be that verbal psychophysical reports of stimuli in this part of the visual field depend on the integrity of V2 and V3 as well as V1 (which appears to be functionally intact). It should also be added that DF has a known lesion in anterior V1 in the left hemisphere, resulting in a partial quadrantanopia. That region of damage, which can be viewed in Figure 3, lies in cortex anterior to the cortical locations activated here with the retinotopic mapping procedure. Therefore, any reduction in activation is not likely to be related to this damage.

Activation patterns to disparity, motion, objects, and color Using the retinotopic mapping stimuli, the majority of activation was in the ventral visual regions. To determine whether this pattern of activation was consistent across stimulus types, the patterns of activation to a variety of stimuli were investigated. Figure 4 shows the whole-brain activation patterns in response to disparity, motion, objects, and color for DF, an age-matched female control participant, and the group data. The responses of the control group $(n=8)$ are shown in Figure $4 B$. Note that the use of the mixed-effects group analysis reduces the significance of acti- 


\section{Visual angle}

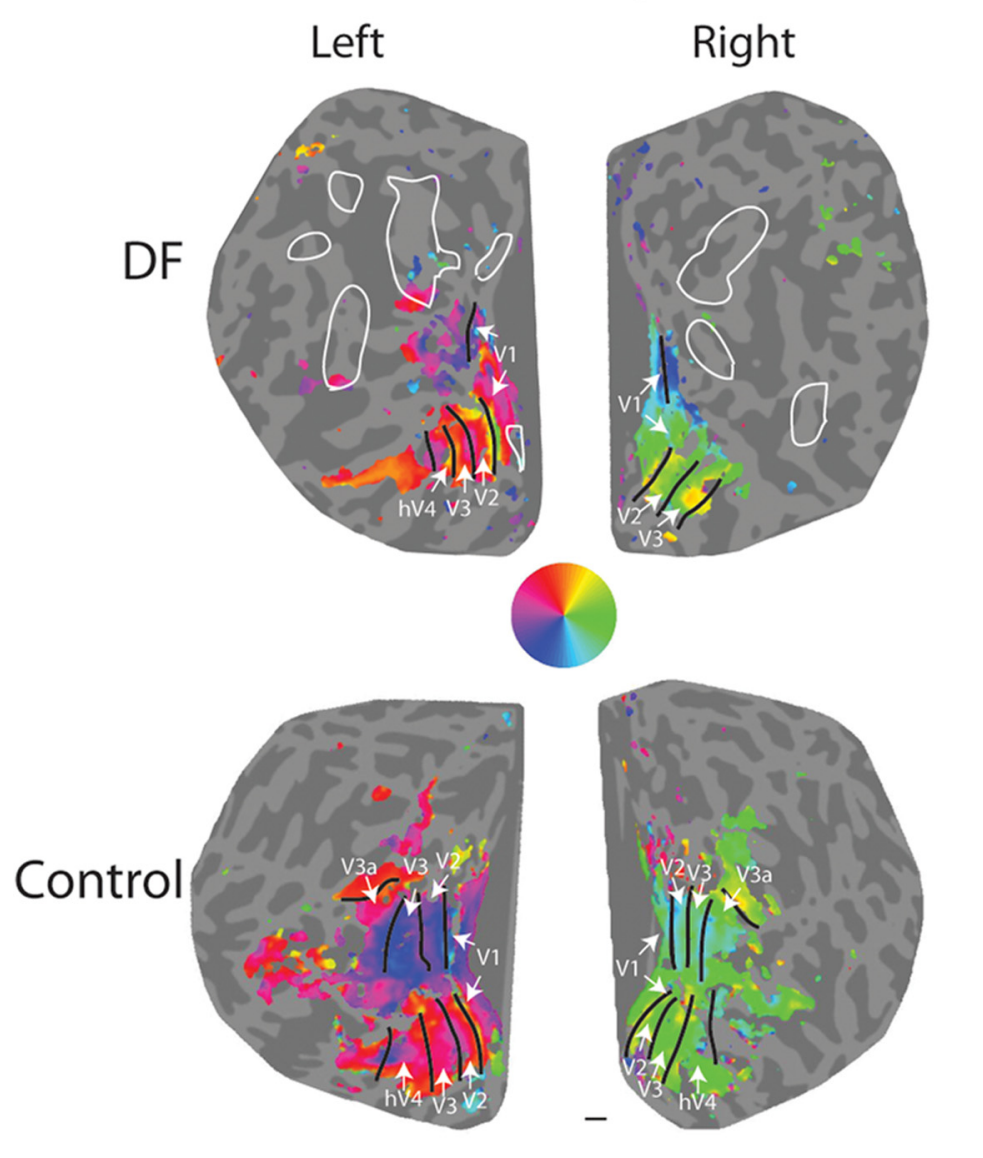

\section{Eccentricity}
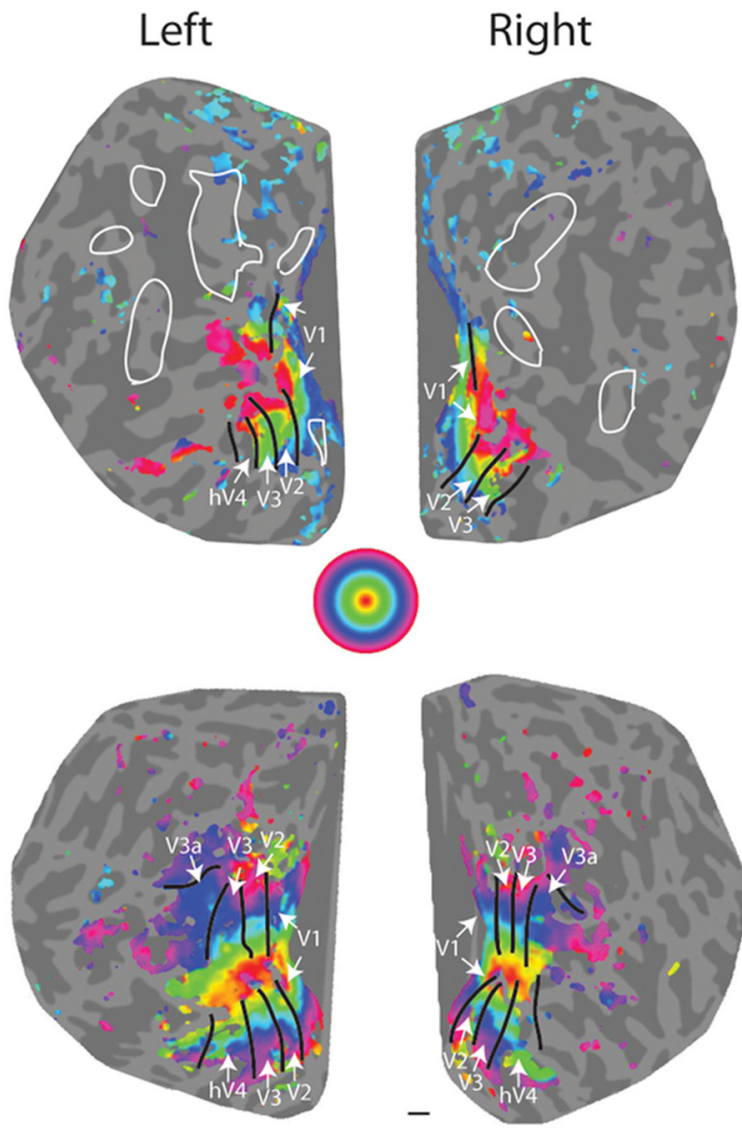



Figure 3. Retinotopic maps of functional activity for patient DF and a control subject shown on flattened representations of the occipital cortex. The mapping in DF was sufficient to define the ventral portions of visual areas V1, V2, V3 (both hemispheres) and hV4 (right hemisphere only). The white arrows in the renderings of DF's brain indicate these visual areas. Responses in the dorsal sections were considerably less clear for both the rotating wedge stimulus used to define angular position (left column) and the expanding wedge stimulus to map eccentricity (right column). The locations and extents of structurally defined lesions of DF's cortex have been transferred from Figure 1 using a criterion of $<1.2$ mm cortical thickness and are shown as white outline contours. The small lesion in the peripheral field representation of ventral V1 in the left hemisphere is devoid of gray matter.

vation because of the interparticipant variability. However, the patterns of activation are similar to those in the literature for similar stimuli (Kourtzi and Kanwisher, 2000; Minini et al., 2010).

\section{DF's cortical response to binocular disparity}

DF was able to describe confidently the disparity-defined checkerboard when shown the stimulus outside of the scanner before the start of the scan session. Interestingly, despite her ability to report her perception of the depth stimulus, the extent of activation throughout her occipital cortex is considerably reduced compared with that of controls. In DF's cortex, there is occipital activation in posterior V1 and the fusiform gyrus, in addition to a small region of the parietal cortex that is activated by all stimulus types in DF but not consistently in control participants (the MNI coordinates for this activation and others are shown in Table 2). Both the age-matched control participant and the control group showed considerably more extensive activation along the ventral surface of the cortex and into the more dorsal occipital regions, such as $\mathrm{V} 3 \mathrm{a}$.

The disparity-related activations analyzed here consist of comparisons between the responses to a disparity-defined checkerboard in contrast to the response to zero-disparity dots. V1 is known to have a large number of neurons tuned to zero disparity
(Poggio et al., 1988; Cumming and DeAngelis, 2001; Prince et al., 2002), so it may be that this choice of comparison is unsuitable for DF because she may rely on the activation in V1. Read et al. (2010) demonstrated that DF can readily detect some forms of stereoscopic disparity, but unlike control participants, her performance does not improve in the presence of a surrounding disparate stimulus, which provides a relative disparity signal. Control participants increased their stereoscopic sensitivity by a factor of 5 when a spatially adjacent reference target was present (Read et al., 2010), but DF did not.

Other evidence suggests that the relevant processing of relative disparity for this type of task takes place in the ventral visual stream (Janssen et al., 1999; Neri et al., 2004; Parker, 2007). In human participants, this is likely to include LOC, and therefore including the relative disparity in the checkerboard may not benefit DF in the way it does control participants. That is, DF may use similar mechanisms for perceiving the flat, zero-disparity plane and the checkerboard stimulus, resulting in no net change in activation. However, it should be noted that DF is able to use disparity very effectively to resolve the direction of rotation of structure-from-motion figures (Read et al., 2010) and that DF's thresholds are such that it is likely that the relevant cue is the relative disparity information between the planes of moving dots. 


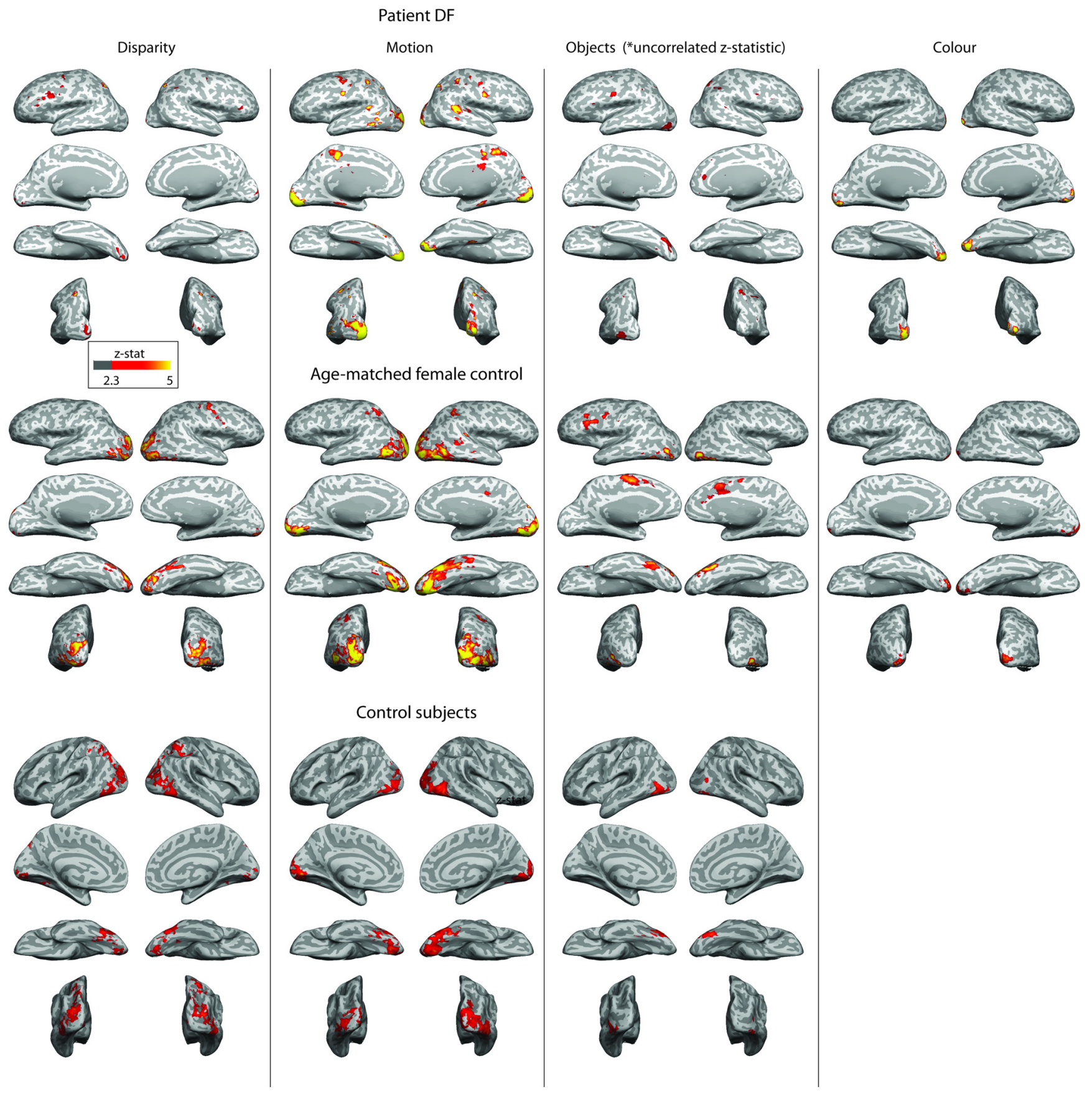

Figure 4. Activation to disparity, motion, object, and color stimuli in patient DF, an age- and gender-matched control participant, and the control group response. The activation to both disparity and objects is considerably reduced in DF. In contrast, the response to motion is extensive and highly significant. Note that, for DF, the cluster correction has not been applied to activations by objects, because this abolished any activation. The significance is lower in the images from the control group, because of the interparticipant variability, but the extent of activation is greater than in DF to all stimuli.

\section{DF's neural responses to motion stimuli}

The activation to motion in DF is comparable with that in individual control participants, with the exception of area MT/V5, which shows very little activity in DF. Although most of the more anterior regions activated in DF are not statistically significant in the group data, the majority of individual participants show the pattern of anterior activation in the region of the parietal operculum and planum temporale, as shown previously (Antal et al., 2008) It is also clear that the early visual area activation is similar to control participants, both the individual shown here and the group.
Interestingly, there have been rather few investigations of motion perception in DF, beyond those performed in the initial testing (Milner et al., 1991; de-Wit et al., 2009). DF is able to distinguish correctly direction of motion of moving random dot patterns correctly when the dot pattern is coherent; as noise is added, reducing the coherence of the motion signal, her performance drops rapidly compared with control subjects. For the current brain-imaging study, we used a fully coherent motion stimulus, so it is perhaps not surprising that there is strong activation to this stimulus. There is extensive activation throughout the cortex in DF, and the change in blood oxygenation level- 
Table 2. Foci of activation to the disparity, object, and color stimuli in patient DF

\begin{tabular}{lll}
\hline Region & Coordinates & z-Statistic \\
\hline Disparity & & \\
$\quad$ Posterior V1 & $(18,-100,6)$ & 3.4 \\
& $(-8,-100,6)$ & 3.0 \\
$\quad$ Ventral occipital & $(-14,-84,-16)$ & 4.1 \\
$\quad$ Frontal & $(-48,0,34)$ & 4.7 \\
Objects $\star$ & & \\
LOC & $(-36,-84,-4)$ & 3.4 \\
& $(54,-66,4)$ & 2.6 \\
Parietal & $(-32,-70,52)$ & 5.0 \\
& $(22,-72,50)$ & 4.1 \\
Color & $(-12,-86,-22)$ & \\
Fusiform & $(18,-82,-12)$ & 5.2 \\
& $(-38,-84,-22)$ & 5.6 \\
Lateral & $(36,-84,-16)$ & 5.2 \\
Posterior V1 & $(-4,-104,0)$ & 4.6 \\
& $(12,-98,0)$ & 4.8 \\
\hline
\end{tabular}

The $\star$ symbol next to the activation to "Objects" indicates that these activation peaks were extracted from results that were not cluster corrected.

dependent (BOLD) response is significantly greater in DF's V1 than the control participants.

However, an unexpected finding was the lack of evidence for activation in DF's area MT/V5, an area believed to be critically important for motion perception. Zihl et al. (1983) described a patient with damage to this area who was motion blind, although it may be that, in their patient, the affected areas were more extensive. It may be that this weak or absent activation in MT/V5 in DF could account for the lack of robust motion perception as coherence is reduced, a phenomenon paralleled by changes in performance after lesions of MT/V5 in the macaque monkey (Newsome and Paré, 1988).

The human MT/V5 complex is now characterized as comprising at least two temporo-occipital areas (TO1 and TO2) that border the lateral occipital areas (LO1 and LO2) (Amano et al., 2009). The weaker functional response of the V5/MT complex, particularly in the right hemisphere, may reflect the damage in the immediately neighboring regions of LOC. Notably the cortical pathway from V1 to V5/MT passes under the LOC in the human brain (Song et al., 2003). The activation in DF's cortex in the region of the parietal operculum in the right hemisphere appears to be enhanced compared with the left (\%BOLD change, right, $0.47 ;$ left, -0.1 ). In contrast, the older control participants showed the opposite pattern (right, $0.02 \pm 0.19$; left, $0.29 \pm$ 0.39 ), although there is considerable intersubject variability and the difference is not significant. The increased response in the right hemisphere of DF may reflect a compensatory use of this cortical area for motion processing after the damage to more posterior areas.

\section{Neural responses to color and objects}

Responses to colored Mondrian patterns compared with blackand-white versions are shown for DF and the age-matched control. No responses are shown for the control group, because there was considerable variability in the response of control participants and no significant group response. In contrast, the response of DF to the colored stimuli extended into the fusiform gyrus, as reported previously (James et al., 2003; Cavina-Pratesi et al., 2010a,b).

The considerable reduction in activation to complete objects compared with scrambled versions of those objects is exemplified by the absence of any cluster-corrected significant activation in DF. In Figure 4, the activation patterns are shown as raw, uncorrected $z$-statistics and are still very restricted in both extent and significance. The left hemisphere shows a small patch of activation in LOC, with no occipital activity in the right hemisphere.

\section{Quantification of BOLD responses to localizer stimuli}

Quantitative measures of percentage BOLD change to all four localizer stimuli are presented on flattened cortex in Figure 5, where they can be related to the previously defined functional retinotopic divisions of early cortical areas and the extent of structural lesions of DF's cortex. Numerical values for the activations to disparity, motion, and colored objects were extracted from the retinotopically defined regions $\mathrm{V} 1, \mathrm{~V} 2$, and $\mathrm{V} 3 \mathrm{a}$, in addition to MT/V5 and LOC (Fig. 6). In each graph, error bars show SDs across control participants. The asterisks indicate that DF's responses were significantly lower than the control mean $(p<0.05)$, and the plus symbols indicate that her responses were significantly greater than the control mean $(p<0.05)$ using the CGP-modified $t$ test.

Consistent with the information provided by the retinotopic maps, these measures indicate that, in DF, there is considerable activation of the early visual areas, particularly V1 and V2. Most notable is the significantly greater response to motion stimuli in DF's V1 compared with control participants. This provides evidence that V1 is intact. Although V2 shows some reduction in cortical thickness and a lack of retinotopy in the dorsal portion, the BOLD response to motion is still comparable with control participants. In contrast, responses in $\mathrm{V} 3 \mathrm{a}$ are reduced relative to controls, even in response to motion, as might be predicted by the lack of dorsal activation in the retinotopic maps. The centers of gravity of these activations are summarized in MNI coordinates in Table 2.

Although the "motion" data shown in the figures are from the group of eight control subjects, additional analysis restricted to the five age-matched controls was also performed. Interestingly, the percentage BOLD change in this older group is higher in early visual areas (V1, $1.4 \pm 0.25$, not significant; V2, $1.5 \pm 0.11, t=$ 4.1, $p<0.05$; V3a, $1.61 \pm 0.11, t=9.7, p \ll 0.001$; MT, $0.96 \pm$ $0.19, t=4.1, p<0.05$; LOC, $0.72 \pm 0.07, t=4.8, p<0.01$; all $t$ tests). Contrary to expectations, there was no reduction in BOLD activity in the older controls. The difference in activation in V1 and V2 between the young and older control groups can be explained by a reduction in negative BOLD surrounding the activated region. This has been described previously in primary somatosensory cortex in older healthy subjects (Gröschel et al., 2013).

\section{Networks involving object perception have decreased white matter integrity}

To examine the connections between LOC and other visual areas, several combinations of connectivity were explored. Fractional anisotropy (FA) and mean diffusivity (MD) were used as quantitative measures of white matter integrity. Probabilistic tractography was run between the following areas: V1 and MT, V1 and V3a, V1 and LOC, and MT and LOC. Additionally, given the widespread nature of the carbon monoxide damage, the corticospinal tract (CST) within DF was exploited to provide a white matter tract as a comparison. This enables the specificity of white matter damage to be evaluated and to reveal whether damage to white matter in DF is primarily located in anatomical connections known to be important for vision. Figure 7 (left) shows the FA in the left and right tracts of DF compared with the mean 


\section{DF activation}
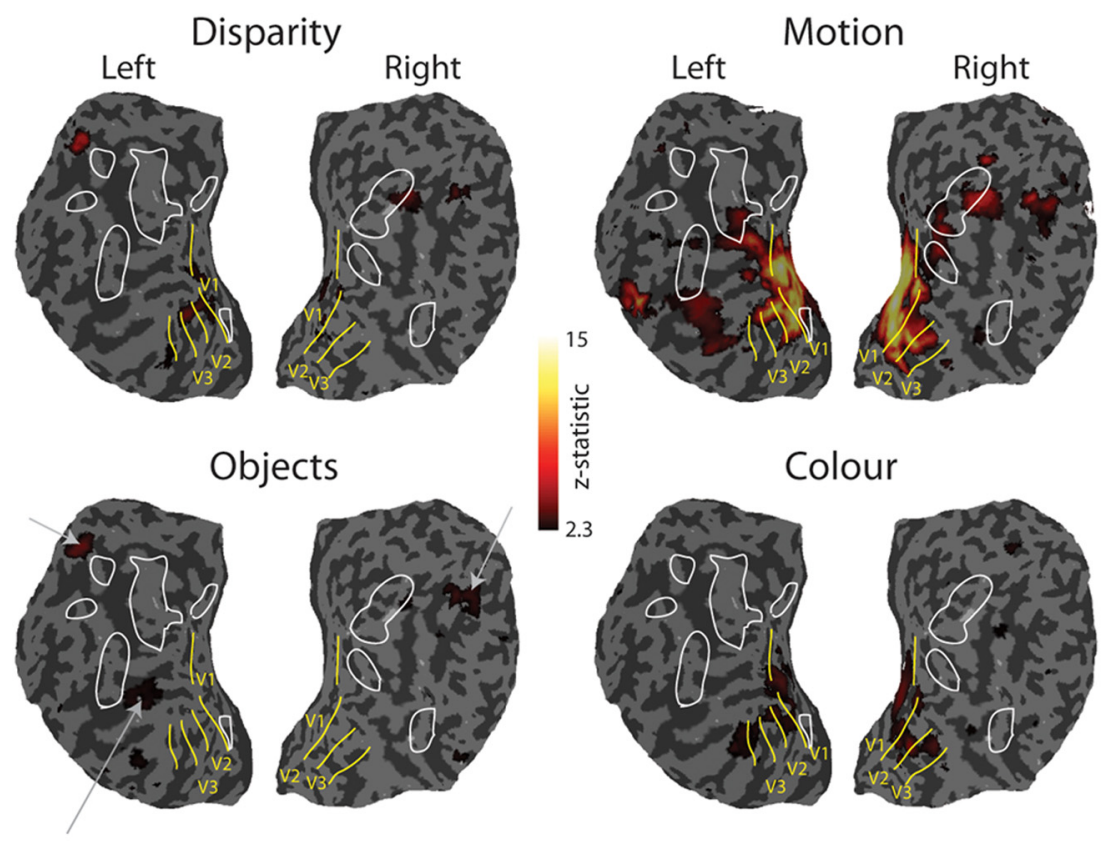

\section{Visual area definitions in inflated space}

\section{DF visual areas}
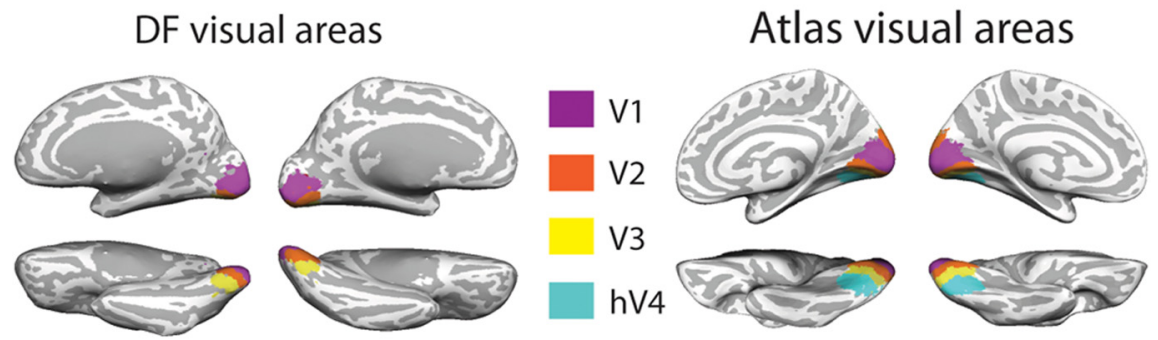

Figure 5. Top panel shows activations of DF's occipital cortex to motion-defined, disparity-defined, object, and color stimuli displayed on flattened cortical surfaces with retinotopically defined visual areas and extent of structural lesions overlaid. Extent of $z$-scaled BOLD activation is color coded according to the key (corrected for multiple comparisons), apart from activations to the object stimuli, which are indicated by white arrows pointing to the sites of activations shown in Figure 4, third column (which for DF were uncorrected for multiple observations). Bottom panel shows summary definitions of early visual cortical areas on inflated brain surfaces for comparison between this figure and Figure 4 for DF and from the atlas-based definitions of visual cortical areas for normal brains.

value in the control participants. The right side of the figure shows the MD in the same tracts. Statistical tests on these differences are summarized in Table 3.

Given the results of the cortical thickness analysis and the functional activation to motion stimuli, it is not surprising that the white matter tract between V1 and MT in DF appears not to differ from that for control participants. Consistent with previous studies (Acosta-Cabronero et al., 2010), we find that degeneration affects $\mathrm{MD}$ to a greater extent than FA. It also appears that white matter in the right hemisphere is more severely affected than in the left. This is reflected in the BOLD activation to disparity and objects in DF's brain in Figure 4 (top row), which appear to be greater in the left hemisphere.

The application of diffusion tractography requires careful consideration of its accuracy between the short distances that span the visual areas, in particular between MT and LOC. Much previous work on white matter integrity has focused on major subcortical-cortical (Behrens et al., 2007) or corticocortical tracts. Previous studies of tracts within the occipital cortex have shown that even the very short connections between MT, LOC, and fusiform face area (FFA) could be distinguished in three of four subjects (Kim et al., 2006). For this study, tracts were checked in native space to ensure that the paths were as predicted, before the extraction of the FA and MD values. The values for FA and $\mathrm{MD}$ are weighted by the probability density of the tract; despite all these checks, it is generally likely that shorter tracts will have greater error and/or variability than the major fiber bundles usually studied with diffusion tractography.

\section{Discussion}

Our aim was a comprehensive overview of changes in structure, function, and connectivity in the brain of the visually agnosic patient DF. Despite extensive damage, regions outside occipital cortex appear essentially intact, apart from previously noted damage in the parietal cortex (James et al., 2003). Somatosensory regions are not thinner than agematched and young controls. Indeed, DF's area $3 \mathrm{~b}$ appears significantly thicker than controls, which may reflect increased dependence on touch to recognize objects. Consistent with the lack of general white matter damage, there appears to be no decrease in integrity of the CST.

\section{Cortical thickness reduction is related to decreases in tract integrity}

In patient $\mathrm{DF}$, neither V1 nor MT is thinner than normal. The white matter connecting these two regions shows little difference in integrity compared with both age-matched and young controls. In contrast, gray matter in LOC is thinner in $\mathrm{DF}$, and tracts that run to LOC show significantly decreased integrity, with decreased FA and increased MD. This white matter damage may be a direct consequence of the initial insult or potentially attributable to retrograde degeneration of the tracts innervating LOC. There is evidence in monkeys and humans for loss of white matter integrity transneuronally after visual cortex damage (Weller et al., 1981; Cowey et al., 1999; Bridge et al., 2011), so retrograde degeneration is certainly possible. Equally, if some cortical areas had been initially compromised immediately after the initial accident, they may by now have become reestablished through a variety of possible mechanisms.

Responses in the early visual areas are generally preserved Extensive regions of the early visual areas of DF's cortex are fairly normal: in the ventral portion of the occipital lobe, it is possible to define retinotopic areas $\mathrm{V} 1, \mathrm{~V} 3, \mathrm{~V} 3$, and $\mathrm{hV} 4$. Reasons for the lack of response in the dorsal regions of the occipital cortex are un- 
clear and are not necessarily related to DF's visual agnosia. The retinotopic map in the dorsal portion of V1 is clear, so it may be that the slightly greater reduction in cortical thickness in the dorsal divisions of $\mathrm{V} 2$ and $\mathrm{V} 3$ has affected the BOLD response to a greater extent.

\section{LOC responses are reduced but not abolished in DF}

Although both the gray matter of LOC and the adjacent white matter are severely compromised in DF's brain, small areas of LOC appear to activate to the presentation of object stimuli. These regions are detectable with our protocols, but only if we do not apply cluster correction to the data. This is likely to be attributable to the reduced extent of activation in DF compared with controls. DF can use the color of objects to assist with recognition (GrillSpector et al., 2000), so the color in the object stimuli may increase either the intensity or extent of the BOLD signal (or both) in LOC relative to black-and-white line drawings (James et al., 2003). Moreover, the sensitivity of scanning technology has improved substantially in the past 10 years, mainly as a result of improvements in radio frequency coil design. More detailed measurements in DF may reveal that these portions of LOC with surviving responsiveness are differentially activated by different types of visual stimuli (faces, objects, scenes) as found in intact human cortex (Grill-Spector and Malach, 2004; Sayres and Grill-Spector, 2008).

\section{Involvement of ventromedial areas in the emergence of visual-form agnosia}

On the basis of a case of bilaterally localized stroke that resulted in visual-form agnosia, Karnath et al. (2009) suggested that compromise of function in areas other than the LOC might be responsible for the pattern of defects in patient DF. The structural imaging in DF does not support this view. There is little change in cortical thickness in the ventromedial areas of the occipital cortex, whereas the LOC clearly exhibits adverse changes in DF. There is also evidence that ventromedial areas can be functionally activated in DF, notably in response to the visual motion stimuli.

The identification of an ischemic area on a structural MRI (Karnath et al., 2009) is a different form of test compared with the detailed quantification of changes in cortical thickness that we have undertaken here. On balance, the evidence favors an interpretation of DF's deficit as consistent with compromise of the LOC, which is also the area identified in normal human cortex as involved in visual object recognition (Grill-Spector et al., 2001).

However, the category specificity within the human (and nonhuman primate) visual system is far from absolute. A comparison of activation patterns to faces and objects in the ventral visual stream indicates that there are regions of the ventromedial visual cortex that respond more strongly to objects than faces (Humphrey et al., 1994; Malach et al., 1995). It may well be that bilateral

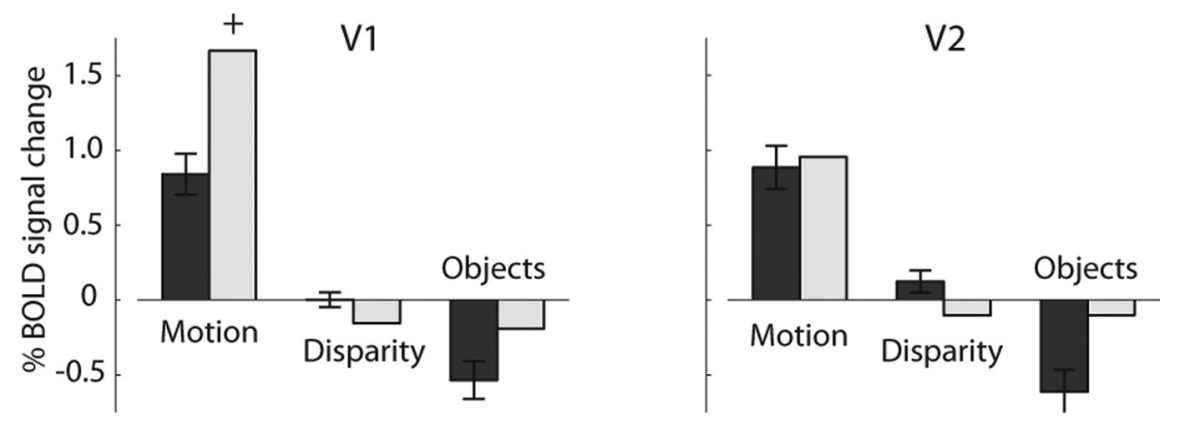

MT
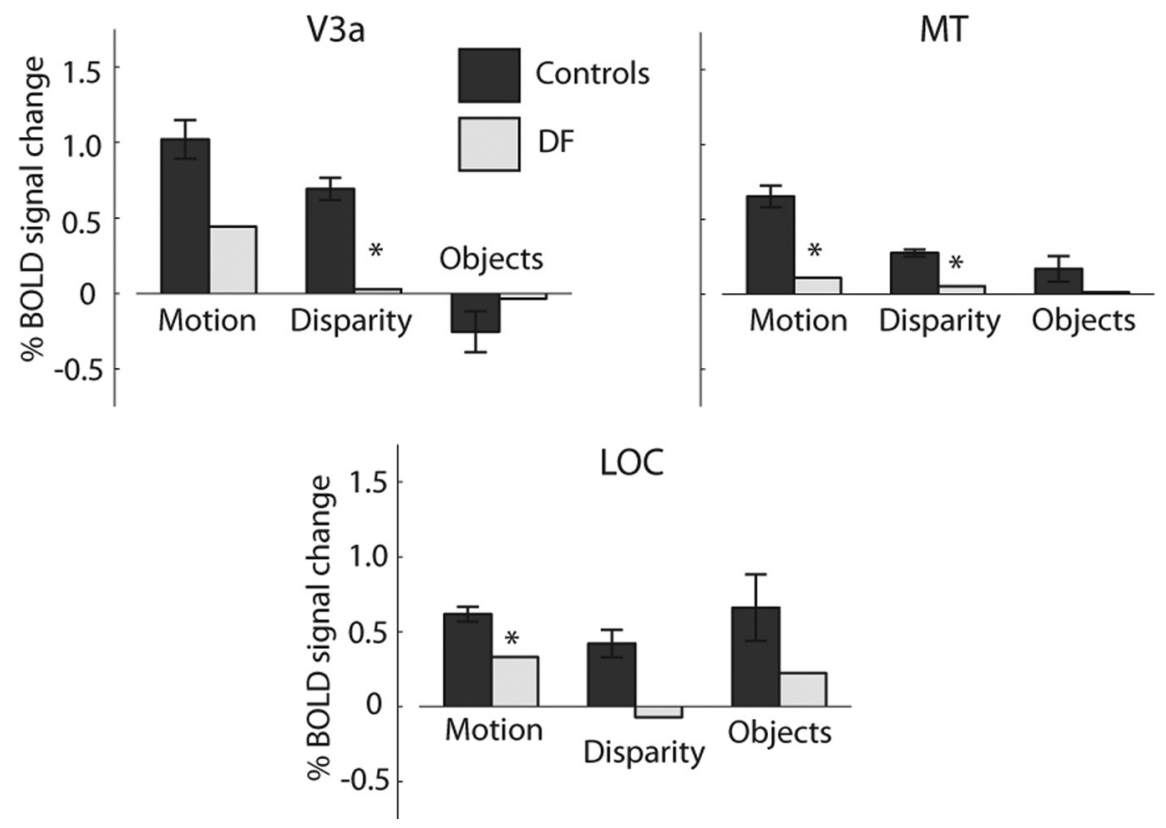

V3a

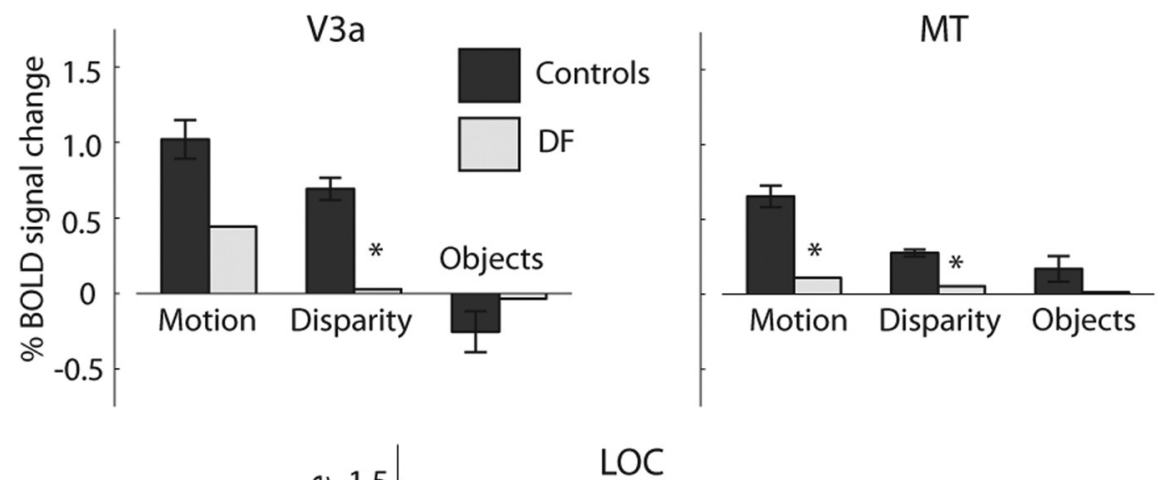

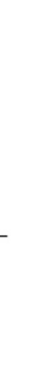

Figure 6. Summary of activations to motion-defined, disparity-defined, and object stimuli in control participants and DF. Areas showing activity significantly lower than the control mean (CGP-modified $t$ test, $p<0.05$ ) are shown by *. The activity to motion stimuli in V1 is significantly greater in DF than the control mean (CGP-modified $t$ test, $p<0.05$ ), denoted by $\dagger$.

damage to either LOC or ventromedial cortex can lead to agnosia and furthermore that the comparison of behavior between these different underlying lesions may shed light on the difference between these object representations.

Our evidence of spared ventromedial and compromised ventrolateral portions of DF's visual cortex is fully in line with previous functional MRI findings showing clear activations for category-specific stimuli only if they activate the more ventromedial areas. Indeed, DF exhibits significant activation for faces in the FFA (Steeves et al., 2006) for color in the anterior collateral sulcus (CoS) (Cavina-Pratesi et al., 2010a) and for texture in the posterior CoS (Cavina-Pratesi et al., 2010a,b).

\section{Structural measures do not necessarily correspond with function}

The structural data indicate that areas V1 and MT are least affected in DF's brain, because both the cortical thickness and the integrity of the white matter between the areas are preserved. BOLD responses to motion are extensive but do not include area MT/V5. One possibility for the lack of activation in area MT/V5 could be a decrease in the motion input via V3. This area appears to show a bias toward "dorsal" type stimuli (Lyon and Connolly, 2012), including considerable numbers of disparity-selective neurons in the macaque (Felleman and Van Essen, 1987; Gegen- 



Figure 7. Measurements of white matter integrity in diffusion-weighted images. FA is significantly decreased particularly in the right hemisphere of $D F$. The left hemisphere shows very little difference from control participants in any of the pathways from V1. Only the pathway between MT and LOC is significantly affected in the left hemisphere, and it was not possible to define a tract in the right hemisphere. The increase in $M D$ reflects the changes in $F A$, with very little difference in the left hemisphere, except for the MT-LOC connection. The asterisks indicate tracts in which the tract integrity is significantly different in DF compared with controls (GGPmodified $t$ test, ${ }^{*} p<0.05$ and ${ }^{* *} p<0.001$ ). The (GP-modified $t$ tests were specifically designed for comparing a single case study against a group of control participants (Crawford et al., 2010).

furtner et al., 1997) and responses to second-order motion in humans (Smith et al., 1998).

One clear conclusion from the new MRI evidence we present here is that one cannot account for the pattern of perceptual and behavioral deficits just by looking at the structural or the functional MRI data taken separately on their own. This is particularly relevant when damage is not restricted to a single brain region, as in toxic damage or some types of trauma to the human brain. For these cases, it is important to apply methods that combine both structural and functional evidence.

\section{The pathway to parietal cortex}

This study has emphasized the widespread damage to the occipital lobe in visual agnosia patient DF while highlighting those regions that are minimally affected: V1, MT/V5, and ventromedial cortex. It is remarkable that DF has many preserved features of her vision; most notably, DF's visually guided locomotion and actions are surprisingly normal behaviorally (Milner et al., 1991; Goodale et al., 1994). Neuroimaging (James et al., 2003) has
Table 3. Statistical tests of differences in diffusion tensor imaging measurements between DF's brain and controls

\begin{tabular}{lcl}
\hline Connection & CGP-modified $t$ statistic & Significance \\
\hline FA V1-MT left & & \\
V1-MT right & -0.73 & NS \\
V1-V3a left & -1.96 & NS \\
V1-V3a right & -0.30 & NS \\
V1-LOC left & -1.17 & NS \\
V1-LOC right & -0.93 & NS \\
MT-LOC left & -3.40 & $p<0.05$ \\
MT-LOC right & -6.20 & $p<0.005$ \\
MT-STS left & None & \\
MT-STS right & +0.56 & NS \\
MD & -0.51 & NS \\
V1-MT left & & \\
V1-MT right & +0.84 & NS \\
V1-V3a left & +0.84 & NS \\
V1-V3a right & -1.72 & NS \\
V1-LOC left & +2.64 & $p<0.058$ \\
V1-LOC right & +1.31 & NS \\
MT-LOC left & +4.70 & $p<0.05$ \\
MT-LOC right & +107.4 & $\mathrm{P}<<0.001$ \\
MT-STS left & None & \\
MT-STS right & -0.69 & NS \\
\hline
\end{tabular}

Values are shown separately for measurements of FA and MD. The CGP-modified $t$ test was specifically designed for comparing a single case study against a group of control participants (Crawford et al., 2010). STS, Superior temporal sulcus.

shown activation in dorsal stream areas, such as human anterior intraparietal area hAIP, during visually guided behavior.

Our more precise measurements of function and structure in DF's occipital lobe raise the question of how visual information might be conveyed to the parietal lobe to guide everyday manual behavior (Culham et al., 2006). Very relevant to the understanding of recovery after brain damage is whether the flow of information in DF's brain through to the parietal cortex follows the same pathways used in the intact, undamaged brain.

Recent research suggests that V1, V2, V3, V3A, MT, and MST are reciprocally connected with area V6 located nearby the parietal occipital sulcus (Galletti et al., 2001) In turn, V6 is connected to areas located in the intraparietal sulcus (IPS) region (the lateral, medial, and ventral intraparietal areas) along with the ventral portion of V6A, located anteriorly to V6, in the parietal lobe. Given the role played by these intraparietal regions in the visual control of eye movements, reaching, and grasping (Grefkes and Fink, 2005) and V6A in reaching (Galletti et al., 2003; CavinaPratesi et al., 2010c; Filimon, 2010), V6 represents a major gateway for distributing visual information arriving from the occipital cortex to the sensorimotor areas in the parietal lobe.

In the present study, we were unable to map the dorsal portions of early visual areas beyond V1 and parts of V3A. However, information could still pass from DF's spared ventral V2 and V3 and areas MT/MST, to area V6, and thence to V6A and intraparietal cortex. Conversely, in line with previous observations (James et al., 2003), our cortical thickness analyses revealed thinned gray matter around the parietal-occipital cortex, in which V6 is located in humans (Pitzalis et al., 2006). To fully elucidate the functioning of DF's dorsal visual stream, future studies will need to investigate the spatial organization of V6 and other parietal areas, such as IPS1-IPS5 (for review, see Silver and Kastner, 2009). 


\section{References}

Acosta-Cabronero J, Williams GB, Pengas G, Nestor PJ (2010) Absolute diffusivities define the landscape of white matter degeneration in Alzheimer's disease. Brain 133:529-539. CrossRef Medline

Amano K, Wandell BA, Dumoulin SO (2009) Visual field maps, population receptive field sizes, and visual field coverage in the human MT+ complex. J Neurophysiol 102:2704-2718. CrossRef Medline

Antal A, Baudewig J, Paulus W, Dechent P (2008) The posterior cingulate cortex and planum temporale/parietal operculum are activated by coherent visual motion. Vis Neurosci 25:17-26. CrossRef Medline

Behrens TE, Berg HJ, Jbabdi S, Rushworth MF, Woolrich MW (2007) Probabilistic diffusion tractography with multiple fibre orientations: What can we gain? Neuroimage 34:144-155. CrossRef Medline

Bondok AA, Sansone FM (1984) Retrograde and transganglionic degeneration of sensory neurons after a peripheral nerve lesion at birth. Exp Neurol 86:322-330. CrossRef Medline

Bridge H (2011) Mapping the visual brain: how and why. Eye (Lond) 25: 291-296. CrossRef Medline

Bridge H, Parker AJ (2007) Topographical representation of binocular depth in the human visual cortex using fMRI. J Vis 7(14):15 11-14. CrossRef

Bridge H, Jindahra P, Barbur J, Plant GT (2011) Imaging reveals optic tract degeneration in hemianopia. Invest Ophthalmol Vis Sci 52:382-388. CrossRef Medline

Castiello U, Jeannerod M (1991) Measuring time to awareness. Neuroreport 2:797-800. CrossRef Medline

Cavina-Pratesi C, Kentridge RW, Heywood CA, Milner AD (2010a) Separate channels for processing form, texture, and color: evidence from FMRI adaptation and visual object agnosia. Cereb Cortex 20:2319-2332. CrossRef Medline

Cavina-Pratesi C, Kentridge RW, Heywood CA, Milner AD (2010b) Separate processing of texture and form in the ventral stream: evidence from FMRI and visual agnosia. Cereb Cortex 20:433-446. CrossRef Medline

Cavina-Pratesi C, Monaco S, Fattori P, Galletti C, McAdam TD, Quinlan DJ, Goodale MA, Culham JC (2010c) Functional magnetic resonance imaging reveals the neural substrates of arm transport and grip formation in reach-to-grasp actions in humans. J Neurosci 30:10306-10323. CrossRef Medline

Cowey A, Stoerig P, Williams C (1999) Variance in transneuronal retrograde ganglion cell degeneration in monkeys after removal of striate cortex: effects of size of the cortical lesion. Vision Res 39:3642-3652. CrossRef Medline

Crawford JR, Garthwaite PH, Porter S (2010) Point and interval estimates of effect sizes for the case-controls design in neuropsychology: rationale, methods, implementations, and proposed reporting standards. Cogn Neuropsychol 27:245-260. CrossRef Medline

Culham JC, Cavina-Pratesi C, Singhal A (2006) The role of parietal cortex in visuomotor control: what have we learned from neuroimaging? Neuropsychologia 44:2668-2684. CrossRef Medline

Cumming BG, DeAngelis GC (2001) The physiology of stereopsis. Annu Rev Neurosci 24:203-238. CrossRef Medline

Dale AM, Fischl B, Sereno MI (1999) Cortical surface-based analysis. I. Segmentation and surface reconstruction. Neuroimage 9:179-194. CrossRef Medline

de-Wit LH, Kentridge RW, Milner AD (2009) Shape processing area LO and illusory contours. Perception 38:1260-1263. CrossRef Medline

Felleman DJ, Van Essen DC (1987) Receptive field properties of neurons in area V3 of macaque monkey extrastriate cortex. J Neurophysiol 57:889920. Medline

Filimon F (2010) Human cortical control of hand movements: parietofrontal networks for reaching, grasping, and pointing. Neuroscientist 16:388407. CrossRef Medline

Fischl B, Dale AM (2000) Measuring the thickness of the human cerebral cortex from magnetic resonance images. Proc Natl Acad Sci U S A 97: 11050-11055. CrossRef Medline

Fischl B, van der Kouwe A, Destrieux C, Halgren E, Ségonne F, Salat DH, Busa E, Seidman LJ, Goldstein J, Kennedy D, Caviness V, Makris N, Rosen B, Dale AM (2004) Automatically parcellating the human cerebral cortex. Cereb Cortex 14:11-22. CrossRef Medline

Galletti C, Gamberini M, Kutz DF, Fattori P, Luppino G, Matelli M (2001) The cortical connections of area V6: an occipito-parietal network processing visual information. Eur J Neurosci 13:1572-1588. CrossRef Medline
Galletti C, Kutz DF, Gamberini M, Breveglieri R, Fattori P (2003) Role of the medial parieto-occipital cortex in the control of reaching and grasping movements. Exp Brain Res 153:158-170. CrossRef Medline

Gegenfurtner KR, Kiper DC, Levitt JB (1997) Functional properties of neurons in macaque area V3. J Neurophysiol 77:1906-1923. Medline

Goodale MA, Milner AD (1992) Separate visual pathways for perception and action. Trends Neurosci 15:20-25. CrossRef Medline

Goodale MA, Milner AD, Jakobson LS, Carey DP (1991) A neurological dissociation between perceiving objects and grasping them. Nature 349: 154-156. CrossRef Medline

Goodale MA, Meenan JP, Bülthoff HH, Nicolle DA, Murphy KJ, Racicot CI (1994) Separate neural pathways for the visual analysis of object shape in perception and prehension. Curr Biol 4:604-610. CrossRef Medline

Grefkes C, Fink GR (2005) The functional organization of the intraparietal sulcus in humans and monkeys. J Anat 207:3-17. CrossRef Medline

Grill-Spector K, Malach R (2004) The human visual cortex. Annu Rev Neurosci 27:649-677. CrossRef Medline

Grill-Spector K, Kushnir T, Hendler T, Malach R (2000) The dynamics of object-selective activation correlate with recognition performance in humans. Nat Neurosci 3:837-843. CrossRef Medline

Grill-Spector K, Kourtzi Z, Kanwisher N (2001) The lateral occipital complex and its role in object recognition. Vision Res 41:1409-1422. CrossRef Medline

Gröschel S, Sohns JM, Schmidt-Samoa C, Baudewig J, Becker L, Dechent P, Kastrup A (2013) Effects of age on negative BOLD signal changes in the primary somatosensory cortex. Neuroimage 71:10-18. CrossRef Medline

Hesse C, Ball K, Schenk T (2012) Visuomotor performance based on peripheral vision is impaired in the visual form agnostic patient DF. Neuropsychologia 50:90-97. CrossRef Medline

Humphrey GK, Goodale MA, Jakobson LS, Servos P (1994) The role of surface information in object recognition: studies of a visual form agnosic and normal subjects. Perception 23:1457-1481. CrossRef Medline

James TW, Culham J, Humphrey GK, Milner AD, Goodale MA (2003) Ventral occipital lesions impair object recognition but not object-directed grasping: an fMRI study. Brain 126:2463-2475. CrossRef Medline

Janssen P, Vogels R, Orban GA (1999) Macaque inferior temporal neurons are selective for disparity-defined three-dimensional shapes. Proc Natl Acad Sci U S A 96:8217-8222. CrossRef Medline

Karnath HO, Rüter J, Mandler A, Himmelbach M (2009) The anatomy of object recognition-visual form agnosia caused by medial occipitotemporal stroke. J Neurosci 29:5854-5862. CrossRef Medline

Kim M, Ducros M, Carlson T, Ronen I, He S, Ugurbil K, Kim DS (2006) Anatomical correlates of the functional organization in the human occipitotemporal cortex. Magn Reson Imaging 24:583-590. CrossRef Medline

Kourtzi Z, Kanwisher N (2000) Cortical regions involved in perceiving object shape. J Neurosci 20:3310-3318. Medline

Lyon DC, Connolly JD (2012) The case for primate V3. Proc Biol Sci 279: 625-633. CrossRef Medline

Malach R, Reppas JB, Benson RR, Kwong KK, Jiang H, Kennedy WA, Ledden PJ, Brady TJ, Rosen BR, Tootell RB (1995) Object-related activity revealed by functional magnetic-resonance-imaging in human occipital cortex. Proc Natl Acad Sci U S A 92:8135-8139. CrossRef Medline

Milner AD (1997) Vision without knowledge. Philos Trans R Soc Lond B Biol Sci 352:1249-1256. CrossRef Medline

Milner AD, Perrett DI, Johnston RS, Benson PJ, Jordan TR, Heeley DW, Bettucci D, Mortara F, Mutani R, Terazzi E, et al. (1991) Perception and action in "visual form agnosia." Brain 114:405-428. CrossRef

Minini L, Parker AJ, Bridge H (2010) Neural modulation by binocular disparity greatest in human dorsal visual stream. J Neurophysiol 104: 169-178. CrossRef Medline

Neri P, Bridge H, Heeger DJ (2004) Stereoscopic processing of absolute and relative disparity in human visual cortex. J Neurophysiol 92:1880-1891. CrossRef Medline

Newsome WT, Paré EB (1988) A selective impairment of motion perception following lesions of the middle temporal visual area (MT). J Neurosci 8:2201-2211. Medline

O'Donnell P, Buxton PJ, Pitkin A, Jarvis LJ (2000) The magnetic resonance imaging appearances of the brain in acute carbon monoxide poisoning. Clin Radiol 55:273-280. CrossRef Medline

Parker AJ (2007) Binocular depth perception and the cerebral cortex. Nat Rev Neurosci 8:379-391. CrossRef Medline

Pitzalis S, Galletti C, Huang RS, Patria F, Committeri G, Galati G, Fattori P, 
Sereno MI (2006) Wide-field retinotopy defines human cortical visual area v6. J Neurosci 26:7962-7973. CrossRef Medline

Poggio GF, Gonzalez F, Krause F (1988) Stereoscopic mechanisms in monkey visual cortex: binocular correlation and disparity selectivity. J Neurosci 8:4531-4550. Medline

Prince SJ, Pointon AD, Cumming BG, Parker AJ (2002) Quantitative analysis of the responses of $\mathrm{V} 1$ neurons to horizontal disparity in dynamic random-dot stereograms. J Neurophysiol 87:191-208. Medline

Radoeva PD, Prasad S, Brainard DH, Aguirre GK (2008) Neural activity within area $\mathrm{V} 1$ reflects unconscious visual performance in a case of blindsight. J Cogn Neurosci 20:1927-1939. CrossRef Medline

Read JC, Phillipson GP, Serrano-Pedraza I, Milner AD, Parker AJ (2010) Stereoscopic vision in the absence of the lateral occipital cortex. PLoS One 5:e12608. CrossRef Medline

Sayres R, Grill-Spector K (2008) Relating retinotopic and object-selective responses in human lateral occipital cortex. J Neurophysiol 100:249-267. CrossRef Medline

Silver MA, Kastner S (2009) Topographic maps in human frontal and parietal cortex. Trends Cogn Sci 13:488-495. CrossRef Medline

Smith AT, Greenlee MW, Singh KD, Kraemer FM, Hennig J (1998) The processing of first- and second-order motion in human visual cortex assessed by functional magnetic resonance imaging (fMRI). J Neurosci 18:3816-3830. Medline

Song AW, Harshbarger T, Li T, Kim KH, Ugurbil K, Mori S, Kim DS (2003) Functional activation using apparent diffusion coefficient-dependent contrast allows better spatial localization to the neuronal activity: evidence using diffusion tensor imaging and fiber tracking. Neuroimage 20:955-961. CrossRef Medline

Steeves JK, Culham JC, Duchaine BC, Pratesi CC, Valyear KF, Schindler I, Humphrey GK, Milner AD, Goodale MA (2006) The fusiform face area is not sufficient for face recognition: evidence from a patient with dense prosopagnosia and no occipital face area. Neuropsychologia 44:594-609. CrossRef Medline

Wandell BA, Winawer J (2011) Imaging retinotopic maps in the human brain. Vision Res 51:718-737. CrossRef Medline

Weller RE, Kaas JH, Ward J (1981) Preservation of retinal ganglion cells and normal patterns of retinogeniculate projections in prosimian primates with long-term ablations of striate cortex. Invest Ophthalmol Visual Sci 20:139-148. Medline

Zihl J, von Cramon D, Mai N (1983) Selective disturbance of movement vision after bilateral brain damage. Brain 106:313-340. CrossRef Medline 